\title{
Recombinant E2 glycoprotein of bovine viral diarrhea virus induces a solid humoral neutralizing immune response but fails to confer total protection in cattle
}

S. Chimeno Zoth ${ }^{1,2}$,

M.R. Leunda ${ }^{3}$,

A. Odeón ${ }^{3}$ and

O. Taboga ${ }^{1,2}$
${ }^{1}$ Institute of Biotechnology CICVyA, INTA, Castelar, Buenos Aires, Argentina ${ }^{2}$ CONICET, Buenos Aires, Argentina

${ }^{3}$ INTA EEA Balcarce, Balcarce, Buenos Aires, Argentina
Correspondence

S. Chimeno Zoth

Instituto de Biotecnología

CICVyA, INTA

Castelar, CC25 (1712)

Buenos Aires

Argentina

Fax: +54-011-4621-0199

E-mail: schimeno@cnia.inta.gov.ar

Research supported by PICT (No. 08-00067-01290) from FONCyT,

Argentina.

....................

Received September 12, 2006 Accepted March 26, 2007 .......................

\section{Abstract}

Two recombinant baculoviruses were produced in order to obtain a bovine viral diarrhea virus (BVDV) immunogen: AcNPV/E2 expressing E2 glycoprotein, and AcNPV/E0E1E2 expressing the polyprotein region coding for the three structural proteins of BVDV (E0, E1, and E2). Mice were immunized with Sf9 cells infected with the recombinant baculoviruses in a water in oil formulation and the production of neutralizing antibodies was evaluated. Since E2 elicited higher neutralizing antibody titers than E0-E1-E2 polyprotein, it was selected to immunize cattle. Calves received two doses of recombinant E2 vaccine and were challenged with homologous BVDV 37 days later. The recombinant immunogen induced neutralizing titers which showed a mean value of $1.5 \pm 0.27$ on the day of challenge and reached a top value of $3.36 \pm 0.36,47$ days later ( 84 days post-vaccination). On the other hand, sera from animals which received mock-infected Sf9 cells did not show neutralizing activity until 25 days post-challenge (62 days post-vaccination), suggesting that these antibodies were produced as a consequence of BVDV challenge. Even when no total protection was observed in cattle, in vitro viral neutralization assays revealed that the recombinant immunogen was able to induce neutralizing antibody synthesis against the homologous strain as well as against heterologous strains in a very efficient way.

Bovine viral diarrhea virus (BVDV) is an important pathogen of cattle worldwide, generating considerable economic losses for the livestock industry. BVDV belongs to the genus Pestivirus, within the family Flaviviridae (1) and is classified into biotypes and genotypes based on the cytopathic effect on
Key words

- Bovine viral diarrhea virus

- Recombinant E2 glycoprotein cell culture and on nucleic acid differences, respectively (2). The high antigenic diversity associated with BVDV genotypes imposes a critical constraint on the development of efficacious vaccines to control the disease (3). BVDV is a small positivestranded RNA virus with a genome of ap- 
proximately $12.5 \mathrm{~kb}$ coding for a polyprotein which is co- and post-translationally processed by either host or viral proteases, yielding at least 12 individual proteins, three of which are structural glycoproteins: E0, E1, and E2 (4). Glycoprotein E2 is the most immunogenic protein of BVDV and elicits high titers of neutralizing antibodies after infection (5). Since virus transmission can be blocked in the presence of neutralizing antibodies, E2 constitutes an excellent candidate for the formulation of a recombinant vaccine (6).

Both attenuated and killed BVDV vaccines are available. Nevertheless, in spite of the efforts made in this field, an effective and safe BVDV vaccine has not yet been obtained. The development of a subunit vaccine with advantages in terms of biosecurity and absence of reversion risk would be of great help in formulating strategies for BVDV control. In addition, the use of subunit vaccines as marker vaccines would permit the differentiation of vaccinated from infected animals by a simple serological assay and therefore would be of increased benefit (7).

The aim of the present study was to evaluate the serological response induced by a recombinant immunogen based on Sf9 insect cells expressing E2 glycoprotein of the NADL strain of BVDV.

E2 and E0-E1-E2-coding regions were amplified from cDNA synthesized from genomic RNA of the NADL strain of BVDV (ATCC) as described previously (8), using primers E2f (5' GGGATCCACCATGGT ACAGGGCATTCTG 3') and E2r (5' GAA GCTTCTAGATTAGAGTAAGACCC ACTT 3') for E2 glycoprotein and E0polf (5'CGCGGATCCATGAAACTGGAAAAA GCATTG 3') and E2polr (5' AAACTGCA GTTAGAGTAAGACCCACTT 3') for the polyprotein. The amplified fragments were cloned into the pVL1393 transfer plasmid (Pharmingen, San Diego, CA, USA) to obtain pVLE2 and pVLE0E1E2 plasmids. Recombinant baculoviruses (AcNPV/E2 and
AcNPV/E0E1E2) were obtained by standard procedures (9). For antigen production, a previously described protocol was used (8). Briefly, monolayers of Sf9 cells were infected with each viral inoculum at a multiplicity of infection of 5 . After $72 \mathrm{~h}$ at $27^{\circ} \mathrm{C}$, cells were collected and centrifuged for 15 $\mathrm{min}$ at $200 \mathrm{~g}$. Then, cells were washed with PBS, pH 6.2, and resuspended in cracking buffer. Cell extracts were analyzed by SDSPAGE and the presence of recombinant proteins was demonstrated by Western blot.

In order to compare the immunogenicity of both antigens, five BALB/c adult mice were inoculated intraperitoneally with the recombinant immunogen. The inoculum consisted of two doses of $2 \times 10^{7} \mathrm{Sf9}$ cells infected with the recombinant baculovirus (AcNPV/E2 or AcNPV/E0E1E2). Briefly, Sf9 cells grown in T-75 flasks (NUNC, Rochester, NY, USA) were infected with AcNPV/ E2 or AcNPV/E0E1E2 as mentioned above. After incubation, the infected cells were collected, pelleted, washed, and resuspended in PBS at a concentration of $2 \times 10^{7}$ cells $/ 100$ $\mu \mathrm{L}$. Each dose consisted of $100 \mu \mathrm{L}$ of the cell suspension emulsified in $100 \mu \mathrm{L}$ oil adjuvant (10). Inoculations were performed on days 0 and 14. Sera collected 21 days after immunization were analyzed for the presence of neutralizing antibodies against the homologous BVDV NADL strain. Viral neutralization assays were performed in MDBK cells using standard microtitration procedures (11) and titers were determined by the method of Reed and Muench (12). Figure 1 shows that both recombinant immunogens elicited high titers of neutralizing antibodies in comparison with the negative control (mice immunized with mock-infected Sf9 cells formulated with the same adjuvant). The recombinant vaccine based on E2 glycoprotein alone proved to be significantly $(\mathrm{P}<$ 0.01 ) more efficient in the induction of the neutralizing response than the other one, based on the polyprotein (E0-E1-E2). For this reason, the immunogen based on $\mathrm{E} 2$ was 
selected for cattle vaccination experiments.

The recombinant immunogen for cattle was obtained by a procedure similar to that previously described, i.e., Sf9 cells were grown in spinner flasks containing $1 \times 10^{6}$ cells/mL, cells were infected with AcNPV/ E2 and finally resuspended in PBS at a concentration of $1 \times 10^{8}$ cells $/ \mathrm{mL}$. The presence of E2 antigen was confirmed by Western blot (Figure 2A). Anti-E2 mAb revealed the presence of a major band of about $53 \mathrm{kD}$ in Sf9 cells infected with AcNPV/E2, which corresponded to the molecular weight predicted for the E2 glycoprotein. An additional band of approximately $100 \mathrm{kD}$, compatible with the molecular weight predicted for the homodimeric form (E2-E2), was also observed, in agreement with previous reports (13). The same pattern was observed in Western blots developed with polyclonal sera obtained from BVDV-infected calves, suggesting that the antigenic properties of the recombinant E2 protein are similar to those of viral E2 which elicited the production of these antibodies (data not shown). Furthermore, by treatment with tunicamycin, the recombinant protein was shown to be $\mathrm{N}$ glycosylated in a way similar to that described by Collett et al. (4) when BVDV infected its host cells (data not shown).

Each dose of the recombinant immunogen consisted of $2 \mathrm{~mL}$ of the cell suspension (containing approximately $50 \mu \mathrm{g}$ recombinant E2, as estimated by Western blot) and 2 $\mathrm{mL}$ oil adjuvant. Mock-infected Sf9 cells were processed identically and formulated to be used as negative control. Six British crossbred calves received two doses of the E2 recombinant immunogen and 2 calves received the control immunogen on days 0 and 21 . On day 37 , calves were intranasally challenged with $2.5 \times 10^{7} \mathrm{TCID}_{50}$ of the NADL strain of BVDV. Serum samples obtained periodically were evaluated by a viral neutralization assay (11).

As shown in Figure 2B, all the animals immunized with the recombinant vaccine de- veloped a specific humoral immune response due to vaccination, as indicated by the presence of neutralizing antibodies against the homologous NADL strain at 37 days postvaccination (the day of challenge). In contrast, sera from animals which received mock-infected Sf9 cells did not show neutralizing activity until 25 days post-challenge ( $62 \mathrm{dpv}$ ), suggesting that these antibodies were produced as a consequence of BVDV challenge. After challenge, animals treated with the recombinant vaccine showed a large increase in virus neutralizing titer values, which increased from 1.76 (at $52 \mathrm{dpv}$ ) to 3.36 (at $84 \mathrm{dpv}$ ). In the middle of this period, at 62 day post-vaccination, the mean value was 2.17 for the recombinant vaccine group, as opposed to only 0.9 for the control group.

In order to investigate if antibodies elicited by the recombinant E2 immunogen, which belongs to genotype Ia, cross-neutralized BVDV of different genotypes, viral neutralization assays were also performed against BVDV $1 b$ and BVDV 2.

As shown in Figure 2B, when serum samples were tested against the BVDV 00693 isolate (genotype 1b) (Odeón A, personal communication), the rate of appearance in vivo of neutralizing antibodies obtained was very similar to that observed

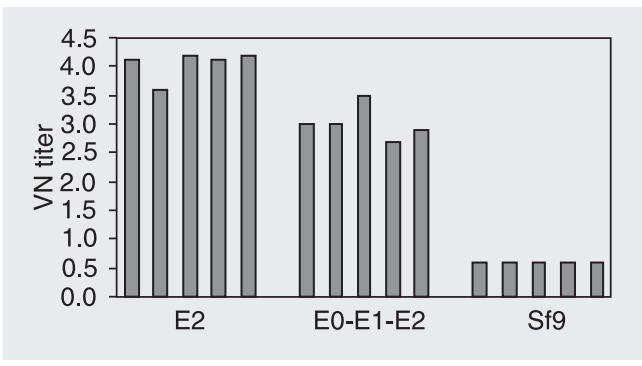

Figure 1. Virus neutralizing (VN) titers against BVDV NADL strain obtained in mice. The presence of neutralizing activity was determined on day 21 , one week after the last immunization. Results were analyzed statistically by one-way ANOVA and means were compared by the Bonferroni test using the Statistix 7.0 Version ${ }^{\odot}$ 1985, 2000 Analytical Software. The differences observed among the three groups were statistically significant $(P<0.01)$. Neutralizing titers were determined by the method of Reed and Muench (12). 
A

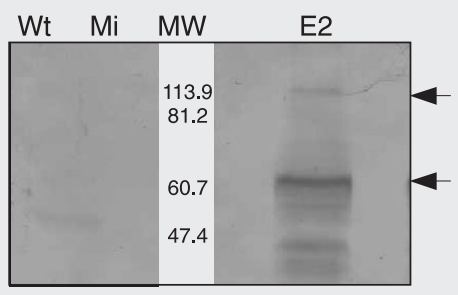

anti-E2 mAb
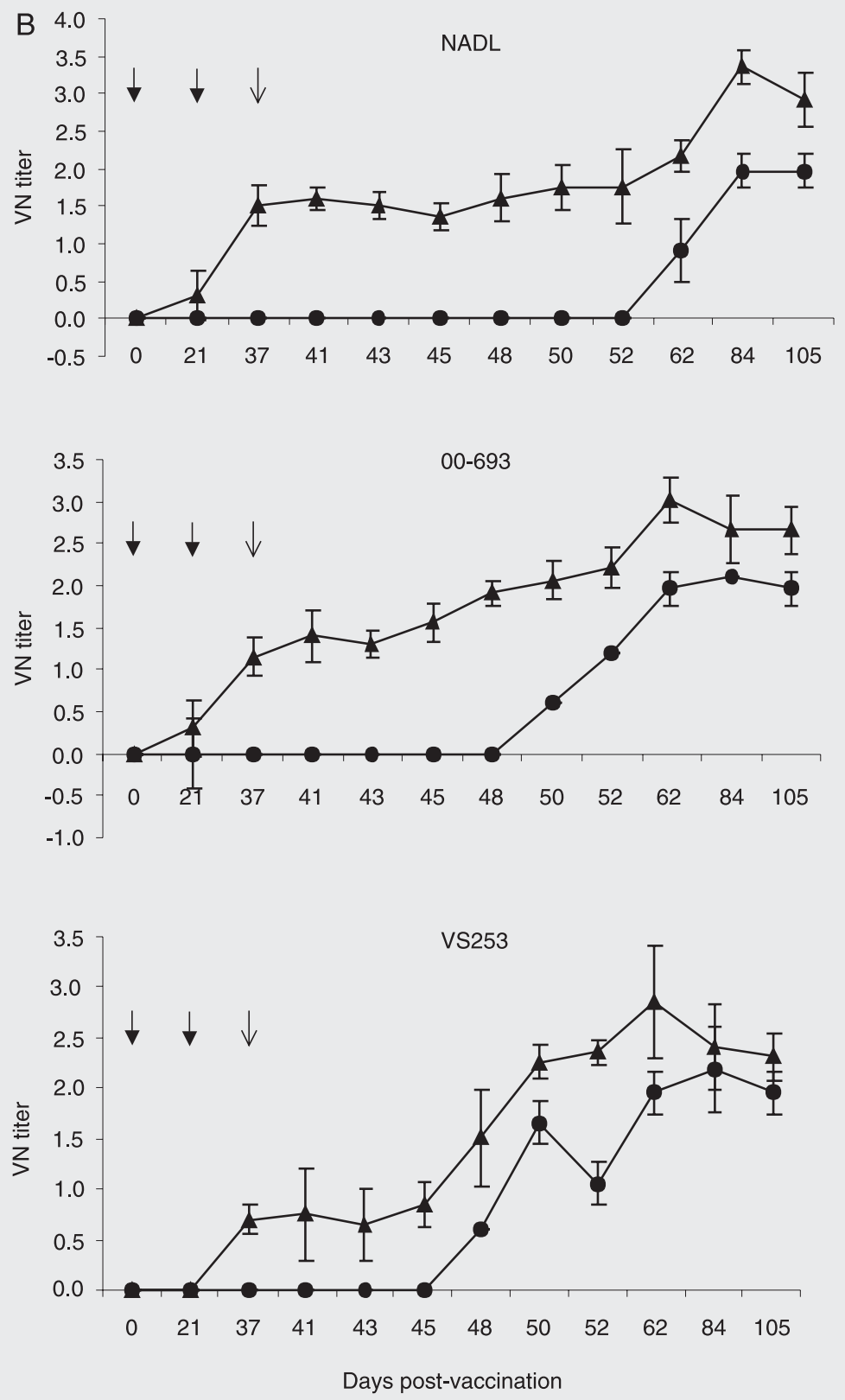

Figure 2. Detection of recombinant E2 protein and evaluation of the neutralizing response induced by the immunization with the recombinant antigen. $A$, Total proteins of extracts of Sf9 cells infected with the recombinant baculovirus AcNPV/E2 were separated by SDSPAGE and the recombinant protein was detected by Western blot using monoclonal antibodies (19f9fb, NADL-E2 specific, diluted 1:10,000, kindly given by Dr. Rubén Donis, Nebraska University, USA). Wt $=$ Sf9 cells infected with wild-type baculovirus AcNPV; $\mathrm{Mi}=$ mock-infected Sf9 cells; MW = molecular weight standards. $B$, Mean virus neutralization (VN) titers of cattle sera against NADL, 00-693 and VS253 strains. The presence of neutralizing activity was determined by VN assays at the time of immunization, of challenge and up to 105 days post-vaccination. The thick arrows indicate vaccination and booster and the light arrow indicates challenge. Neutralizing titers were determined by the method of Reed and Muench (12). The triangles indicate E2 recombinant vaccine and the circles uninfected Sf9 cells.

against the NADL homologous strain.

Finally, serum samples were evaluated against the BVDV VS253 strain (genotype 2; USDA, Ames, IA, USA). On the day of virus challenge, only 2 of the 6 animals from the recombinant vaccine group had seroconverted, and 11 days later (48 days post-vaccination) all the animals in this group showed neutralizing antibodies against BVDV 2 (Figure 2B). Neutralizing titers induced by the recombinant E2 vaccine were higher than those observed in the control group, not only against the homologous strain, but also against heterologous ones, such as the 00-693 isolate (genotype 1b) and VS253 strain (genotype 2). The viral neutralization assay demonstrated that the recombinant immunogen induced a neutralizing response that showed cross-reactivity with BVDV subtype $1 \mathrm{~b}$ and, although to a lesser extent, with BVDV genotype 2, as shown by the results obtained on the day of challenge, when the neutralizing antibodies detected were exclusively induced by the experimental immunogen. Nevertheless, the recombinant immunogen did not completely prevent clinical symptomatology (pyrexia, leukopenia) or viral replication after challenge. Viral isolation assays were performed on samples from ocu- 
lar swabs (collected at $0,4,6,8,11,13$, and 15 days post-challenge) passaged four times in MDBK cells. Viral antigens were detected by immunofluorescence using an anti-BVDV antibody conjugated with FITC (American BioResearch Labs., Seivierville, TN, USA) and the results obtained were confirmed by RT-PCR, as previously described (8) using IA and IB oligonucleotides (IA: 5' GAGGCTAG CCATGCCCTTAGT 3', IB: 5' TCAACTCCA TGTGCCATGTACAGCA 3'), adapted from Pellerin et al. (14), which amplify the fragment comprised between positions 98 and 402 of the non-coding 5 ' region reported for the NADL sequence (15). Samples obtained from both animals of the control group (2/2) revealed the presence of BVDV at 4 and 6 days post-challenge. On the other hand, results obtained for samples of animals vaccinated with the recombinant immunogen showed that 4 animals revealed the presence of BVDV at 6 days post-challenge, while the other 2 animals remained negative throughout the study. Therefore, the results obtained in viral isolation assays indicate that the recombinant immunogen elicits only partial protection in terms of viral replication.

This recombinant vaccine demonstrated the ability to elicit an in vitro neutralizing humoral immune response, which did not correlate completely with in vivo protection. This observation suggests that the presence of neutralizing antibodies against E2 is not the only feature required to avoid BVDV replication in cattle. Undoubtedly, other components of the immune response may be involved in avoiding the infection, as reported by others in studies using inactivated and modified live vaccines (16).

Several studies based on vaccination with E2 glycoprotein expressed in different systems have been reported. Although vaccination assays with baculovirus-expressed E2 protein have been reported $(6,17)$, the experimental vaccine employed in all cases consisted of E2 glycoprotein obtained from supernatants of recombinant baculovirus-in- fected cells. However, there are numerous examples of vaccination with infected cells, in which infected insect cells expressing immunodominant proteins of different viruses induced protection against homologous viral challenge $(18,19)$. This constitutes an interesting option because purification methods are not required to obtain the recombinant protein. Moreover, this option provides a hydrophobic surrounding for the antigen which would contribute to the development of the proper immune response (20).

In the present paper, we report the induction of an immune response in cattle immunized with a recombinant vaccine based on Sf9 insect cells displaying E2 glycoprotein of BVDV, as demonstrated by immunofluorescence assays (data not shown). The results obtained suggest that this methodology constitutes a promising approach to be considered in the design of a recombinant antiBVDV vaccine.

Several parameters such as different doses, immunization schemes and routes of antigen administration remain to be evaluated, and a higher number of animals must be analyzed. Nevertheless, this recombinant vaccine demonstrated the ability to elicit an in vitro neutralizing humoral immune response, not only against the strain from which the antigen was obtained, but also against heterologous BVDV strains.

This vaccine approach represents a very promising tool for the implementation of BVDV eradication campaigns since its use could permit the differentiation between vaccinated and infected animals, since natural infection elicits the production of antibodies against other viral proteins such as E0 and NS3 (8).

\section{Acknowledgments}

The authors are grateful to the veterinarians Eleonora Morrell and Germán Cantón for technical assistance in the assay with the calves. 


\section{References}

1. Horzinek MC. Pestiviruses - taxonomic perspectives. Arch Virol Supp/ 1991; 3: 1-5.

2. Baker JC. The clinical manifestations of bovine viral diarrhea infection. Vet Clin North Am Food Anim Pract 1995; 11: 425-445.

3. Fulton RW, Ridpath JF, Confer AW, Saliki JT, Burge LJ, Payton ME. Bovine viral diarrhoea virus antigenic diversity: impact on disease and vaccination programmes. Biologicals 2003; 31: 89-95.

4. Collett MS, Larson R, Belzer SK, Retzel E. Proteins encoded by bovine viral diarrhea virus: the genomic organization of a pestivirus. Virology 1988; 165: 200-208.

5. Donis RO, Corapi W, Dubovi EJ. Neutralizing monoclonal antibodies to bovine viral diarrhoea virus bind to the $56 \mathrm{~K}$ to $58 \mathrm{~K}$ glycoprotein. J Gen Virol 1988; 69 (Part 1): 77-86.

6. Bolin SR, Ridpath JF. Glycoprotein E2 of bovine viral diarrhea virus expressed in insect cells provides calves limited protection from systemic infection and disease. Arch Virol 1996; 141: 1463-1477.

7. Nettleton PF, Entrican G. Ruminant pestiviruses. Br Vet J 1995; 151 : 615-642.

8. Chimeno Zoth S, Taboga O. Multiple recombinant ELISA for the detection of bovine viral diarrhoea virus antibodies in cattle sera. $J$ Virol Methods 2006; 138: 99-108.

9. O'Reilly D, Miller LK, Luckow VA. Baculovirus expression vectors. A laboratory manual. New York: W.H. Freeman and Company; 1994.

10. Rivenson S, Sadir AM, Gaggino OP, Marcovecchio FE, Zabal O, Laporte O. Estudio comparativo en bovinos de dos vacunas antiaftosa: oleosa e hidroxisaponinada. Rev Med 1982; 63: 364370.

11. Maisonnave J, Rossi CR. A microtiter test for detecting and titrating noncytopathogenic bovine viral diarrhea virus. Arch Virol 1982; 72 : 279-287.
12. Reed LJ, Muench HA. A simple method of estimating fifty percent endpoints. Am J Hyg 1938; 27: 493-497.

13. Weiland E, Stark R, Haas B, Rumenapf T, Meyers G, Thiel HJ. Pestivirus glycoprotein which induces neutralizing antibodies forms part of a disulfide-linked heterodimer. J Virol 1990; 64: 3563-3569.

14. Pellerin $\mathrm{C}$, van den Hurk J, Lecomte J, Tussen P. Identification of a new group of bovine viral diarrhea virus strains associated with severe outbreaks and high mortalities. Virology 1994; 203: 260-268.

15. Colett MS, Larson R, Gold C, Strick D, Anderson DK, Purchio AF. Molecular cloning and nucleotide sequence of the pestivirus bovine viral diarrhea virus. Virology 1988; 165: 191-199.

16. Frey HR, Eicken K, Grummer B, Kenklies S, Oguzoglu TC, Moennig $\mathrm{V}$. Foetal protection against bovine virus diarrhoea virus after twostep vaccination. J Vet Med B Infect Dis Vet Public Health 2002; 49: 489-493.

17. Bruschke CJ, van Oirschot JT, van Rijn PA. An experimental multivalent bovine virus diarrhea virus E2 subunit vaccine and two experimental conventionally inactivated vaccines induce partial fetal protection in sheep. Vaccine 1999; 17: 1983-1991.

18. Wardley RC, Berlinski PJ, Thomsen DR, Meyer AL, Post LE. The use of feline herpesvirus and baculovirus as vaccine vectors for the gag and env genes of feline leukaemia virus. J Gen Virol 1992; 73 (Part 7): 1811-1818.

19. Tami C, Peralta A, Barbieri R, Berinstein A, Carrillo E, Taboga O. Immunological properties of FMDV-gP64 fusion proteins expressed on SF9 cell and baculovirus surfaces. Vaccine 2004; 23: 840-845.

20. Goodman-Snitkoff G, Eisele LE, Heimer EP, Felix AM, Andersen TT, Fuerst TR, et al. Defining minimal requirements for antibody production to peptide antigens. Vaccine 1990; 8: 257-262. 